\title{
HARMONIC HARDY-ORLICZ SPACES
}

\author{
Tero Kilpeläinen, Pekka Koskela and Hiroaki Masaoka
}

\author{
University of Jyväskylä, Department of Mathematics and Statistics \\ P.O. Box 35 (MaD), FI-40014 University of Jyväskylä, Finland; tero.kilpelainen@jyu.fi \\ University of Jyväskylä, Department of Mathematics and Statistics \\ P.O. Box 35 (MaD), FI-40014 University of Jyväskylä, Finland; pekka.j.koskela@jyu.fi \\ University of Jyväskylä, Department of Mathematics and Statistics \\ P.O. Box 35 (MaD), FI-40014 University of Jyväskylä, Finland; hiroaki.h.masaoka@jyu.fi \\ and Kyoto Sangyo University, Faculty of Science, Department of Mathematics \\ Kamigamo-Motoyama, Kitaku, Kyoto 603-8555, Japan; masaoka@cc.kyoto-su.ac.jp
}

\section{Dedicated to Professor Olli Martio on his seventieth birthday.}

\begin{abstract}
Given an open hyperbolic Riemannian manifold, we show that various vector spaces of harmonic functions coincide if and only if they are finite dimensional.
\end{abstract}

\section{Introduction}

In what follows, $R$ will always be an open Riemannian manifold that admits a Green's function. In other words, $R$ is hyperbolic. Let us begin by defining the concept of a harmonic Hardy-Orlicz space on $R$. We consider the class $\mathcal{N}$ of non-negative convex strictly increasing functions $\Phi$ on $[0,+\infty)$ with $\Phi(0)=0$ and $\lim _{t \rightarrow \infty} t^{-1} \Phi(t)=\lim _{t \rightarrow \infty} \Phi(t)=\infty$. Fix $\Phi \in \mathcal{N}$. Then the harmonic Hardy-Orlicz space $H_{\Phi}(R)$ consists of all harmonic functions $u$ for which there exists $\alpha>0$ so that $\Phi(\alpha|u|)$ has a harmonic majorant (i.e. there exist $\alpha>0$ and a harmonic function $v$ such that $\Phi(\alpha|u|) \leq v)$. Notice that the convexity of $\Phi$ guarantees that $H_{\Phi}(R)$ is a vector space. The above definition makes perfect sense also without the assumption that $\lim _{t \rightarrow \infty} t^{-1} \Phi(t)=\infty$. However, if this limit, that exists by convexity of $\Phi$, happened to be bounded, our Hardy-Orlicz space of harmonic functions would simply consist of differences of positive harmonic functions. We refer to [16] for the basic properties of harmonic Hardy-Orlicz spaces.

Our first result shows that the spaces $H_{\Phi}(R)$ and $H_{\Psi}(R)$ may coincide only either when $\Phi$ and $\Psi$ trivially generate the same space or when one (both) of them is finite dimensional.

Theorem 1. Let $R$ be an open hyperbolic Riemannian manifold. Suppose that $\Phi, \Psi \in \mathcal{N}$ with $\lim _{t \rightarrow+\infty} \frac{\Phi(\alpha t)}{\Psi(t)}=+\infty$ for all positive $\alpha$. Then the following three conditions are equivalent:

(i) $H_{\Phi}(R)=H_{\Psi}(R)$,

(ii) $\operatorname{dim}\left(H_{\Phi}(R)\right)<\infty$,

(iii) $\operatorname{dim}\left(H_{\Psi}(R)\right)<\infty$.

doi:10.5186/aasfm.2013.3816

2010 Mathematics Subject Classification: Primary 31C12; Secondary 31C35, 35J70.

Key words: $\mathcal{P}$-Brelot harmonic space, harmonic Hardy-Orlicz space, minimal Martin boundary, harmonic measure. 
Choosing $\Phi(t)=t^{q}$ and $\Psi(t)=t^{p}$ with $1<p<q$ in Theorem 1 allows us to recover the results in [11] for Riemann surfaces.

In the classification theory of Riemann surfaces or Riemannian manifolds, one considers various classes of harmonic functions. Let $H P_{+}(R)$ and $H B_{+}(R)$ be the classes of non-negative harmonic functions and non-negative bounded harmonic functions on $R$, respectively. Denote by $M_{H} B_{+}(R)$ the class of all finite limit functions of monotone increasing sequences of $H B_{+}(R)$. Set

$$
H X(R)=\left\{h_{1}-h_{2}: h_{j} \in H X_{+}(R), j=1,2\right\}
$$

and define $M H B(R)$ analogously. Then, $H B(R)$ is the class of bounded harmonic functions on $R$, and $M H B(R)$ is called the class of quasi-bounded functions on $R$. It is well-known that if $R$ does not admit a Green's function, then $\operatorname{HX}(R)$ $(X=P, B)$ and $M H B(R)$ consist of constant functions (cf. [17]). We always have that $H B(R) \subset H_{\Phi}(R) \subset M H B(R) \subset H P(R)$, and these inclusions can, in general, be strict. Our second result shows that finite dimensionality characterizes the equivalence of a harmonic Hardy-Orlicz space with either of $H B(R), M H B(R)$.

Theorem 2. Let $R$ be an open hyperbolic Riemannian manifold. Suppose that $\Phi \in \mathcal{N}$. Then the following conditions are equivalent:

(i) $H B(R) \supset H_{\Phi}(R)$, and hence, $H B(R)=H_{\Phi}(R)$,

(ii) $M H B(R) \subset H_{\Phi}(R)$, and hence, $M H B(R)=H_{\Phi}(R)$,

(iii) one (all) of $\operatorname{dim}(H B(R)), \operatorname{dim}(M H B(R))$, or $\operatorname{dim}\left(H_{\Phi}(R)\right)$ is finite.

Notice that we have not included the case $H P(R)=H_{\Phi}(R)$ in Theorem 2. The following corollary to Theorem 2 shows that this can only happen when the two dimensions in question are equal. We will provide relevant examples elsewhere. We write $\mathcal{N}_{\infty}$ for the collection of those $\Phi \in \mathcal{N}$ with

$$
\lim _{t \rightarrow+\infty} \frac{\Phi(t)}{t}=+\infty
$$

Corollary 1. Suppose that $\Phi \in \mathcal{N}_{\infty}$. Then the following two conditions are equivalent:

(i) $H P(R) \subset H_{\Phi}(R)$, and hence $H P(R)=H_{\Phi}(R)$,

(ii) $\operatorname{dim} H P(R)=\operatorname{dim} H_{\Phi}(R)<+\infty$.

In fact, the above results extend to a more general setting that includes suitably weighted Riemannian manifolds. In order to single out the properties essential for the above results, we give the proofs below in an axiomatic setting described in Section 2. This setting allows us to produce rather simple examples where all these various classes of harmonic functions coincide. In Section 4, we give three such examples. The crucial tool for us is the concept of minimal Martin boundary that will be described in Section 2.

The paper is organized as follows. Section 2 contains preliminaries and Section 3 the proofs of the above two theorems. In the final section we give three examples.

Acknowledgement. This work was carried out during the third author's visit at the University of Jyväskylä. He would like to express his gratitude to the Department of Mathematics and Statistics for hospitality. 


\section{Preliminaries}

Let us describe the abstract setting. Let $\Omega$ be a locally compact, non-compact, connected and locally connected Hausdorff space. Let $\mathcal{H}$ be a class of real valued continuous functions, called harmonic functions, on open subsets of $\Omega$ satisfying the following three axioms:

Axiom 1. $\mathcal{H}$ forms a linear sheaf.

Axiom 2. There is a base for the topology of $\Omega$ such that each set in the base is a regular domain for $\mathcal{H}$.

Axiom 3. For any domain $U$ in $\Omega$ any ordered increasing directed family of harmonic functions defined on $U$ has an upper envelope which is either $+\infty$ everywhere in $U$ or harmonic in $U$.

A pair $(\Omega, \mathcal{H})$ satisfying the above properties is called a Brelot harmonic space (cf. $[1,3]$ ). Furthermore, we assume that there exists a positive potential on $\Omega$. Such a Brelot harmonic space is called (cf. [3]) a $\mathcal{P}$-Brelot harmonic space. For an open set $U \subset \Omega$, set $\mathcal{H}(U)=\{u \in \mathcal{H}: u$ is harmonic in $U\}$. Throughout this paper we further assume that there exists a countable base for the open sets of $\Omega$ and that the constant function $h(x) \equiv 1$ belongs to $\mathcal{H}(\Omega)$.

For a $\mathcal{P}$-Brelot harmonic space $(\Omega, \mathcal{H})$, we define the various classes of harmonic functions described in the introduction analogously, replacing harmonic functions by elements of $\mathcal{H}$.

Let $S^{+}$be the set of non-negative superharmonic functions on $\Omega$, defined, as usual, via a comparison principle. Denote by $\Delta_{1}^{M}=\Delta_{1}^{\Omega, M}$ the set of extreme harmonic functions (or minimal harmonic functions after Martin) of a compact and metrizable base $\Lambda$ of the cone $S^{+}$. As usual, we call it the minimal Martin boundary of $\Omega$. We refer to $[2,3,5,7]$ for a detailed discussion on the minimal Martin boundary and on the Martin boundary. The intuitive picture to have in mind is to consider points in the boundary of the unit disk both as points and Poisson kernels (associated to these points).

The following Martin representation theorem (cf. $[3,7])$ is the fundamental result regarding Martin boundaries. It also explains why we concentrate on the minimal Martin boundary $\Delta_{1}^{M}$.

Theorem M. For each $u \in H P_{+}(\Omega)$, there exists a unique positive measure $\mu_{u}$ on $\Lambda$ so that $\mu_{u}\left(\Lambda \backslash \Delta_{1}^{M}\right)=0$ and

$$
u(z)=\int_{\Delta_{1}^{M}} h(z) d \mu_{u}(h)
$$

Let $\omega^{M}(\cdot)$ be the Martin representing measure of 1 , that is,

$$
1=\int_{\Delta_{1}^{M}} h(z) d \omega^{M}(h)
$$

for each $z \in \Omega$. In this paper we call $\omega^{M}$ the harmonic measure on $\Delta_{1}^{M}$. Given $z \in \Omega$, set $d \omega_{z}^{M}(\cdot)=h(z) d \omega^{M}(h)$. We call $\omega_{z}^{M}$ the harmonic measure with respect to $z$. Then, for $A \subset \Delta_{1}, \omega^{M}(A)>0$ if and only if $\omega_{z}^{M}(A)>0(z \in \Omega)$.

The following proposition gives us a characterization of $M H B(\Omega)$. 
Proposition 1. ([16, Theorems 5 and 7], cf. [2, Folgesatz 13.1 and Satz 13.4]) The following conditions are equivalent:

(i) $u \in M H B(\Omega)$,

(ii) $u$ has the minimal fine limit $u^{*}(h)$ at almost every point $h \in \Delta_{1}^{M}$ with respect to the harmonic measure and

$$
u(z)=\int_{\Delta_{1}^{M}} u^{*}(h) d \omega_{z}^{M}(h) .
$$

(iii) there exists a Borel function $u^{*}(h)$ on $\Delta_{1}^{M}$ with

$$
u(z)=\int_{\Delta_{1}^{M}} u^{*}(h) d \omega_{z}^{M}(h) .
$$

In the above proposition, by the Martin representation theorem, for any other representation $\int f^{*} d \omega_{z}^{M}$ of $u$, we have that $f^{*}=u^{*}$ on $\Delta_{1}^{M}$ except possibly for a null set with respect to the harmonic measure.

Recall that $H_{\Phi}(\Omega)$ is a linear space and that, if there exist positive constants $\alpha, \beta$ and $t_{0}$ with $\Phi(t) \leq \alpha \Psi(\beta t)$ for all $t \geq t_{0}$, then $H_{\Psi}(\Omega) \subset H_{\Phi}(\Omega)$. Set $H_{\Phi+}(\Omega):=$ $H_{\Phi}(\Omega) \cap H P_{+}(\Omega)$. It is well known that $H_{\Phi}(\Omega)=H_{\Phi+}(\Omega)-H_{\Phi+}(\Omega)$.

Our next proposition provides us with a characterization of $H_{\Phi}(\Omega)$.

Proposition 2. (cf. [16, Theorem 12]) Let $\Phi \in \mathcal{N}_{\infty}$. The following conditions are equivalent:

(i) $u \in H_{\Phi}(\Omega)$,

(ii) $u$ has the minimal fine limit $u^{*}(h)$ at almost every point $h \in \Delta_{1}^{M}$ with respect to the harmonic measure,

$$
u(z)=\int_{\Delta_{1}^{M}} u^{*}(h) d \omega_{z}^{M}(h),
$$

and there exists a positive constant $\alpha$ with

$$
\int_{\Delta_{1}^{M}} \Phi\left(\alpha\left|u^{*}\right|\right) d \omega^{M}<\infty
$$

By the above proposition it is easily seen that $H_{\Phi}(\Omega) \subset M H B(\Omega)$ for all $\Phi \in \mathcal{N}_{\infty}$.

\section{Proofs of our theorems}

We will establish the various equivalences asserted in our theorems via the following property that may or may not hold for a general mimimal Martin boundary. We say that the mimimal Martin boundary $\Delta_{1}^{M}$ is finitely atomic if there exists a null set $N$ of $\Delta_{1}^{M}$ with respect to the harmonic measure so that $\Delta_{1}^{M} \backslash N$ consists of finitely many points, each of positive harmonic measure. Since the harmonic measure of $\Delta_{1}^{M}$ is nonzero, being finitely atomic is actually equivalent to the existence of a null set whose complement has a finite number of elements.

3.1. Proof of Theorem 1. Fix $\alpha>0$, and set $H_{\alpha}(t):=\frac{\Phi(\alpha t)}{\Psi(t)}$. By assumption $\lim _{t \rightarrow+\infty} H_{\alpha}(t)=\infty$. Suppose that (i) holds. Fix a point $z_{0}$ of $\Omega$. First we prove the following subclaim.

Subclaim 1. There exists no sequence $\left\{A_{n}\right\}_{n=1}^{\infty}$ of pairwise disjoint subsets of $\Delta_{1}^{M}$ with $\omega_{z_{0}}^{M}\left(A_{n}\right)>0$ for each $n \geq 1$. 
Proof. Suppose on the contrary that there exists a sequence $\left\{A_{n}\right\}_{n=1}^{\infty}$ of pairwise disjoint subsets of $\Delta_{1}^{M}$ with $\omega_{z_{0}}^{M}\left(A_{n}\right)>0$ for each $n$. Since $\omega_{z_{0}}^{M}\left(\Delta_{1}^{M}\right)=1$, by passing to a subsequence, we may assume that

$$
\omega_{z_{0}}^{M}\left(A_{n}\right) \leq \frac{1}{n^{2} \max \left\{t>0: H_{\alpha} \circ \Psi^{-1}(t)=2^{n}\right\}},
$$

for each $n$. Set

$$
f_{\alpha}^{*}(h)= \begin{cases}\Psi^{-1}\left(\frac{1}{n^{2} \omega_{z_{0}}^{M}\left(A_{n}\right)}\right) & \text { for } h \in A_{n} \\ 0 & \text { for } h \in \Delta_{1}^{M} \backslash \cup_{n=1}^{+\infty} A_{n} .\end{cases}
$$

Then, we have

$$
\int_{\Delta_{1}^{M}} \Psi\left(\left|f_{\alpha}^{*}(h)\right|\right) d \omega_{z_{0}}^{M}(h)=\sum_{n=1}^{\infty} \frac{1}{n^{2} \omega_{z_{0}}^{M}\left(A_{n}\right)} \omega_{z_{0}}^{M}\left(A_{n}\right)=\sum_{n=1}^{\infty} \frac{1}{n^{2}}=\frac{\pi^{2}}{6} .
$$

On the other hand, we have

$$
\begin{aligned}
\int_{\Delta_{1}^{M}} \Phi\left(\alpha\left|f_{\alpha}^{*}(h)\right|\right) d \omega_{z_{0}}^{M}(h) & =\sum_{n=1}^{\infty} \Phi\left(\alpha \Psi^{-1}\left(\frac{1}{n^{2} \omega_{z_{0}}^{M}\left(A_{n}\right)}\right)\right) \omega_{z_{0}}^{M}\left(A_{n}\right) \\
& =\sum_{n=1}^{\infty} \frac{\omega_{z_{0}}^{M}\left(A_{n}\right)}{n^{2} \omega_{z_{0}}^{M}\left(A_{n}\right)} H_{\alpha}\left(\Psi^{-1}\left(\frac{1}{n^{2} \omega_{z_{0}}^{M}\left(A_{n}\right)}\right)\right) \\
& =\sum_{n=1}^{\infty} \frac{1}{n^{2}} H_{\alpha}\left(\Psi^{-1}\left(\frac{1}{n^{2} \omega_{z_{0}}^{M}\left(A_{n}\right)}\right)\right) .
\end{aligned}
$$

Since

$$
\frac{1}{n^{2} \omega_{z_{0}}^{M}\left(A_{n}\right)} \geq \max \left\{t>0: H_{\alpha} \circ \Psi^{-1}(t)=2^{n}\right\}
$$

we conclude that

$$
\int_{\Delta_{1}^{M}} \Phi\left(\alpha\left|f_{\alpha}^{*}(h)\right|\right) d \omega_{z_{0}}^{M}(h) \geq \sum_{n=1}^{\infty} \frac{2^{n}}{n^{2}}=\infty .
$$

Set

$$
f^{*}(h)=\sum_{l=1}^{\infty} \frac{1}{2^{l}} f_{1 / 4^{l}}^{*}(h) \quad\left(h \in \Delta_{1}^{M}\right) \quad \text { and } \quad f(z)=\int_{\Delta_{1}^{M}} f^{*}(h) d \omega_{z}^{M}(h) .
$$

By convexity,

$$
\begin{aligned}
\int_{\Delta_{1}^{M}} \Psi\left(\left|f^{*}(h)\right|\right) d \omega_{z_{0}}^{M}(h) & =\int_{\Delta_{1}^{M}} \Psi\left(\sum_{n=1}^{\infty} \frac{1}{2^{l}} f_{1 / 4^{l}}^{*}(h)\right) d \omega_{z_{0}}^{M}(h) \\
& \leq \int_{\Delta_{1}^{M}}\left[\sum_{l=1}^{\infty} \frac{1}{2^{l}} \Psi\left(f_{1 / 4^{l}}^{*}(h)\right)\right] d \omega_{z_{0}}^{M}(h) \\
& =\sum_{l=1}^{\infty} \frac{1}{2^{l}} \int_{\Delta_{1}^{M}} \Psi\left(f_{1 / 4^{l}}^{*}(h)\right) d \omega_{z_{0}}^{M}(h)=\sum_{l=1}^{\infty} \frac{1}{2^{l}} \frac{\pi^{2}}{6}=\frac{\pi^{2}}{6}<\infty
\end{aligned}
$$

Since

$$
\lim _{t \rightarrow \infty} \frac{\Psi(t)}{t}=\infty
$$


we also have that $\int_{\Delta_{1}^{M}}\left|f^{*}(h)\right| d \omega_{z}^{M}(h)<\infty$. By Proposition 1 we conclude that $f \in M H B(\Omega)$ and that $f$ has the minimal fine limit $f^{*}(h)$ at almost every point $h \in \Delta_{1}^{M}$ with respect to $\omega_{z_{0}}^{M}$. Hence, by Proposition 2, we find that $f \in H_{\Psi}(\Omega)$.

Let $\beta$ be any positive number. Since $\Phi$ is increasing, we may suppose that $\beta<1$. There exists an integer $l_{0}$ with $\frac{1}{2^{l_{0}}} \leq \beta<\frac{1}{2^{l_{0}-1}}$. Hence

$$
\begin{aligned}
\int_{\Delta_{1}^{M}} \Phi\left(\beta\left|f^{*}(h)\right|\right) d \omega_{z_{0}}^{M}(h) & \geq \int_{\Delta_{1}^{M}} \Phi\left(\frac{1}{2^{l_{0}}}\left|f^{*}(h)\right|\right) d \omega_{z_{0}}^{M}(h) \\
& \geq \int_{\Delta_{1}^{M}} \Phi\left(\frac{1}{2^{l_{0}}} \frac{1}{2^{l_{0}}} f_{1 / 4^{l_{0}}}^{*}(h)\right) d \omega_{z_{0}}^{M}(h) \\
& =\int_{\Delta_{1}^{M}} \Phi\left(\frac{1}{4^{l_{0}}} f_{1 / 4^{l_{0}}}^{*}(h)\right) d \omega_{z_{0}}^{M}(h)=\infty .
\end{aligned}
$$

By Proposition 2 we find that $f \in H_{\Psi}(\Omega) \backslash H_{\Phi}(\Omega)$, which contradicts (i), and Subclaim 1 follows.

Subclaim 2. Given a point $h \in \Delta_{1}^{M}$ with $\omega_{z_{0}}^{M}\left(U_{\rho}(h)\right)>0$ for each positive $\rho$, where $U_{\rho}(h)$ is the ball with center $h$ and radius $\rho$ with respect to a metric on $\Lambda$, necessarily $\omega_{z_{0}}^{M}(\{h\})>0$. (Recall that $\Lambda$ is metrizable [7].)

Proof. Indeed, otherwise there is a decreasing sequence $\left\{\rho_{n}\right\}_{n=1}^{\infty}$ with $\lim _{n \rightarrow \infty} \rho_{n}=0$ and $\omega_{z_{0}}^{M}\left(U_{\rho_{n}}(h) \backslash U_{\rho_{n+1}}(h)\right)>0$, which contradicts Subclaim 1.

Relying on Subclaim 1 and Subclaim 2, we now conclude that $\Delta_{1}^{M}$ is finitely atomic. Indeed, define

$$
N=\left\{h \in \Delta_{1}^{M}: \text { there exists a positive } \rho_{h} \text { with } \omega_{z_{0}}^{M}\left(U_{\rho_{h}}(h)\right)=0\right\}
$$

and set $F=\Delta_{1}^{M} \backslash N$. Clearly $F \cup N=\Delta_{1}^{M}, F \cap N=\emptyset$, and, for any $h \in F$, we have that $\omega_{z_{0}}^{M}(\{h\})>0$. Furthermore, $F$ must be a finite set by Subclaim 1. Hence it suffices to prove that $\omega_{z_{0}}^{M}(N)=0$. Set $O=\cup_{h \in N} U_{\rho_{h}}(h)$. Clearly $O$ is an open subset of $\Lambda$ and $O \cap \Delta_{1}^{M}=N$. By the Lindelöf theorem there exists a sequence $\left\{k_{n}\right\}_{n=1}^{\infty}$ in $N$ with $O=\cup_{n=1}^{\infty} U_{\rho_{k_{n}}}\left(k_{n}\right)$. Hence $\omega_{z_{0}}^{M}(N) \leq \omega_{z_{0}}^{M}(O) \leq \sum_{n=1}^{\infty} \omega_{z_{0}}^{M}\left(U_{\rho_{k_{n}}}\left(k_{n}\right)\right)=0$, and we deduce that, $\omega_{z_{0}}^{M}(N)=0$.

In conclusion, we can find a nullset $N$ of $\Delta_{1}^{M}$ with respect to $\omega_{z_{0}}^{M}$ such that $\Delta_{1}^{M} \backslash N$ consists of finitely many points, each of positive harmonic measure. Let $n_{0}$ be the cardinal number of $\Delta_{1}^{M} \backslash N$ and suppose $\Delta_{1}^{M} \backslash N=\left\{h_{1}, \cdots, h_{n_{0}}\right\}$. Fix $u \in M H B(\Omega)$. By Proposition 1, $u$ has the minimal fine limit $u^{*}(h)$ at almost every point $h \in \Delta_{1}^{M}$ with respect to $\omega_{z_{0}}^{M}$ and $u(z)=\int_{\Delta_{1}^{M}} u^{*}(h) d \omega_{z}^{M}(h)$. Hence

$$
u(z)=\sum_{n=1}^{n_{0}} u^{*}\left(h_{n}\right) \omega_{z}^{M}\left(\left\{h_{n}\right\}\right),
$$

with $\left|u^{*}\left(h_{n}\right)\right|<\infty$, for $n=1, \ldots, n_{0}$. This easily yields that $\operatorname{dim}(M H B(\Omega)) \leq n_{0}<$ $\infty$. Since $H_{\Phi}(\Omega) \subset M H B(\Omega)$, we conclude that also $\operatorname{dim}\left(H_{\Phi}(\Omega)\right)<\infty$, and this holds for $H_{\Psi}(\Omega)$ as well. Thus (ii) and (iii) hold.

Suppose then that (ii) or (iii) holds. Since $H B(\Omega) \subset H_{\Phi}(\Omega) \subset H_{\Psi}(\Omega)$ it follows that $\operatorname{dim}(H B(\Omega))<\infty$. From the definition of $M H B(\Omega)$ we conclude that $\operatorname{MHB}(\Omega)=H B(\Omega)$, which guarantees that also $H_{\Phi}(\Omega)=H_{\Psi}(\Omega)$. 
3.2. Proof of Theorem 2. Suppose that (i) holds, that is, $H B(\Omega)=H_{\Phi}(\Omega)$. Fix a point $z_{0} \in \Omega$. We proceed to prove that $\Delta_{1}^{M}$ is finitely atomic. Recall from the proof of Theorem 1 that this follows if we can verify Subclaim 1 from that proof.

Towards this end, we pick a pairwise disjoint subcollection of sets $A_{n} \subset \Delta_{1}^{M}$ with $0<\omega_{z_{0}}^{M}\left(A_{n}\right) \leq 1 / e^{n}$ and set

$$
f^{*}(h)= \begin{cases}\Phi^{-1}(n), & \text { for } h \in A_{n} \\ 0, & \text { for } h \in \Delta_{1}^{M} \backslash \cup_{l=1}^{\infty} A_{n} .\end{cases}
$$

Then, we have

$$
\int_{\Delta_{1}^{M}} \Phi\left(\left|f^{*}(h)\right|\right) d \omega_{z_{0}}^{M}(h)=\sum_{n=1}^{\infty} n \omega_{z_{0}}^{M}\left(A_{n}\right) \leq \sum_{n=1}^{+\infty} \frac{n}{e^{n}}<\infty,
$$

and recalling that $\lim _{t \rightarrow \infty} \frac{\Phi(t)}{t}=\infty$, we find that $\int_{\Delta_{1}^{M}}\left|f^{*}(h)\right| d \omega_{z}^{M}(h)<\infty$. By Proposition 1 and Proposition 2, we have thus constructed an unbounded function $f \in H_{\Phi}(\Omega)$. This contradicts (i).

Suppose then that (ii) holds. Set $H_{\alpha}(t)=\frac{\Phi(\alpha t)}{t}$. We again conclude that $\Delta_{1}^{M}$ is finitely atomic as above, requiring first that

$$
0<\omega_{z_{0}}^{M}\left(A_{n}\right) \leq \frac{1}{n^{3} \max \left\{t>0: H_{\alpha}(t)=2^{n}\right\}},
$$

for each $n \in \mathbf{N}$, and then defining the boundary function by

$$
f_{\alpha}^{*}(h)= \begin{cases}\frac{1}{n^{2} \omega_{z_{0}}^{M}\left(A_{n}\right)}, & \text { for } h \in A_{n}, \\ 0, & \text { for } h \in \Delta_{1}^{M} \backslash \cup_{n=1}^{+\infty} A_{n} .\end{cases}
$$

Set $f^{*}(h)=\sum_{l=1}^{\infty} 1 / 2^{l} f_{1 / 4^{l}}^{*}(h)\left(h \in \Delta_{1}^{M}\right)$ and $f(z)=\int_{\Delta_{1}^{M}} f^{*}(h) d \omega_{z}^{M}(h)$. By similar method to that in the proof of Subclaim 1 of Theorem 1, we find that $f \in M H B(\Omega) \backslash$ $H_{\Phi}(\Omega)$, which contradicts (ii).

Combining the cases above, we have shown that each of (i),(ii) force $\Delta_{1}^{M}$ to be finitely atomic.

Suppose now that $\Delta_{1}^{M}$ is finitely atomic. The argument at the end of the proof of Theorem 1 then guarantees that $\operatorname{dim}(M H B(\Omega))<\infty$, and hence (iii) follows from the inclusions $H B(\Omega) \subset H_{\Phi}(\Omega) \subset M H B(\Omega)$.

We have proven that either of (i) or (ii) imply (iii). Finally, suppose that (iii) holds. Again, the string of inclusion relation from the previous paragraph yields that $\operatorname{dim}(H B(\Omega))<\infty$, and the argument at the very end of the proof of Theorem 1 shows that $H B(\Omega)=M H B(\Omega)$, and consequently, also that $H_{\Phi}(\Omega)=H B(\Omega)$. Thus both (i) and (ii) follow from (iii). This completes our proof.

Remark. It is perhaps worth pointing out that we could further require that the dimensions of our three vector spaces of harmonic functions in Theorem 2 are equal. In fact, it is not hard to verify that this dimension is exactly the number of atoms when $\Delta_{1}^{M}$ is finitely atomic.

3.3. Proof of Corollary 1. Since $H_{\Phi}(\Omega) \subset M H B(\Omega) \subset H P(\Omega)$, (i) is equivalent to $H P(\Omega)=M H B(\Omega)=H_{\Phi}(\Omega)$.

First, we prove that each $h \in \Delta_{1}^{M}$ is a bounded harmonic function and $\omega^{M}(\{h\})>$ 0 . Indeed, each such an $h$ is a minimal non-negative harmonic function and by the 
first paragraph, $h$ is the limit of an increasing sequence of bounded positive harmonic functions $h_{i}$. Now $h_{1} \leq h$ and hence the minimality of $h$ ensures that $h=\alpha h_{1}$ for some positive $\alpha$. Thus $h$ is a bounded harmonic function, and, by Proposition 1, we have the representation

$$
h=\int_{\Delta_{1}^{M}} h^{*}\left(h^{\prime}\right) h^{\prime} d \omega^{M}\left(h^{\prime}\right) .
$$

On the other hand, we also have the representation $h=\int_{\Delta_{1}^{M}} h^{\prime} d \delta_{h}\left(h^{\prime}\right)$, where $\delta_{h}$ is the delta function at $h$. By the uniqueness of the Martin representation, we necessarily have

$$
h^{*}(h) \omega^{M}(\{h\})=1,
$$

whence

$$
\omega^{M}(\{h\})=\frac{1}{h^{*}(h)}>0 .
$$

By Theorem 2 we thus conclude that $\Delta_{1}^{M}$ consists of a finite number of points, each of positive harmonic measure.

Given $u \in H P(\Omega)$, the first paragraph of the proof, Proposition 2 and the second paragraph ensure that

$$
u(z)=\sum_{n=1}^{n_{0}} u^{*}\left(h_{n}\right) \omega_{z}^{M}\left(\left\{h_{n}\right\}\right),
$$

for some $n_{0}$. Hence $\operatorname{dim} H P(\Omega)=\operatorname{dim} H_{\Phi}(\Omega) \leq n_{0}$, and (ii) follows.

Suppose then that (ii) holds. Since $H P(\Omega)$ and $H_{\Phi}(\Omega)$ are linear spaces and $H_{\Phi}(\Omega)$ is a subspace of $H P(\Omega)$, we conclude that $H P(\Omega)=H_{\Phi}(\Omega)$, as desired.

\section{Examples}

In this section we give examples of settings where the minimal Martin boundary consists of a finite number of points, each of positive harmonic measure. For simplicity, we only construct examples, where we have two such points. Our first construction is based on Riemann surfaces, cf. [12], the second one on $\mathbf{R}^{3}$, and the last one on the plane, equipped with a suitable measure.

Example 1. Let $F$ be an open hyperbolic Riemann surface whose minimal Martin boundary is a singleton. A particular case of such a surface is Toki's example [19], cf. [14]. Take a (scaled) copy of the interval $I=[-1,1]$ contained in a chart in $F$. For simplicity, we assume that this copy is $I$. Set $D=F \backslash I$. We take two copies $D_{j}, j=1,2$ of $D$. Joining the upper (resp. lower) edge of $I$ in $D_{1}$ to the lower (resp. upper) edge of $I$ in $D_{2}$, we obtain a 2-sheeted unlimited covering surface $\Omega$ of $D$.

Let $\pi$ be the projection from $\Omega$ onto the base space $F$. We introduce the local coordinates as follows. Let $z$ be a point of $\Omega$. Denote by $B(\pi(z))$ the original local coordinate disk of $\pi(z)$ in $F$. By construction of $F$ (cf. [19]) we remark that $\{z \in \mathbf{C}:|z|<11 / 10\}$ is considered as the original local coordinate disk $B(0)$ of 0 in $F$. We may suppose that, if $\pi(z) \notin I, B(\pi(z) \cap I=\emptyset$. Set

$U(z)= \begin{cases}\text { the component of } \pi^{-1}(B(\pi(z))) \text { containing } z, & \text { if } z \in \Omega \backslash \pi^{-1}(I), \\ \text { the component of } \pi^{-1}\left(B_{\rho(z)}(\pi(z))\right) \text { containing } z, & \text { if } z \in \pi^{-1}(I \backslash\{-1,1\}), \\ \pi^{-1}\left(B_{1 / 20}(\pi(z))\right), & \text { if } z \in \pi^{-1}(\{-1,1\}),\end{cases}$ 
where $\rho(z)=\frac{1}{2} \min \{|\pi(z)-1|,|\pi(z)+1|\}$ and $B_{\rho(z)}(\pi(z))$ is the usual disk with center $\pi(z)$ and radius $\rho(z)$ in $B(0)$. Denote by $\varphi_{x}^{F}$ the original coordinate mapping in $F$. We define the coordinate mapping $\varphi_{z}$ on $U(z)$ by setting

$$
\varphi_{z}(x)= \begin{cases}\varphi_{x}^{F} \circ \pi(x)-\varphi_{x}^{F} \circ \pi(z), & \text { if } z \in \Omega \backslash \pi^{-1}(I), \\ \pi(x)-\pi(z), & \text { if } z \in \pi^{-1}(I \backslash\{-1,1\}), \\ \sqrt{\pi(x)-\pi(z),} & \text { if } z \in \pi^{-1}(\{-1,1\}) .\end{cases}
$$

The above local coordinates give $\Omega$ a conformal structure. Considering the class $\mathcal{H}$ of the usual harmonic functions in open subsets of $\Omega$ with respect to the usual Laplacian on $\Omega$, we easily see that $(\Omega, \mathcal{H})$ is a Brelot harmonic space with a countable base. Moreover, since $\Omega$ is a covering surface of $F$ and $F$ admits a Green's function $G_{z}$ with a pole at $z \in F$, we find that $G_{z} \circ \pi$ is a potential on $\Omega$. Hence $(\Omega, \mathcal{H})$ is $\mathcal{P}$-Brelot harmonic space with a countable base.

Let $\Delta_{1}^{M}$ be the minimal Martin boundary of $\Omega$. Let us check that $\Delta_{1}^{M}$ consists of two points. Towards this end, let $\phi$ be the sheet exchange on $\Omega$, that is, $\phi$ is the transformation on $\Omega$ with $\pi \circ \phi=\pi$ on $\Omega$. Then $\phi \circ \phi$ is the identity mapping on $\Omega$.

Let $h \in \Delta_{1}^{M}$ and set $\hat{h}=h+h \circ \phi$. Since $\hat{h} \circ \phi=\hat{h}$ on $\Omega, \hat{h}$ generates a positive harmonic function on $F$ that we also refer to by $\hat{h}$. Since $F$ does not admit other non-negative harmonic functions than constant functions, there exists a positive number $\alpha$ with $\hat{h}=\alpha$. We remark that there exists a positive constant $\beta$ such that $\beta h \circ \phi \in \Delta_{1}^{M}$. Thus

$$
1=\frac{h}{\alpha}+\frac{h \circ \phi}{\alpha}=\int_{\Delta_{1}^{M}} h d \omega_{1}^{M}(h),
$$

where $\omega_{1}^{M}=\frac{1}{\alpha} \delta_{h}+\frac{1}{\alpha \beta} \delta_{\beta h \circ \phi}$ and $\delta_{h}$ is the Dirac measure at $h \in \Delta_{1}^{M}$, gives us a Martin representation of the constant function 1 . It follows that $\sharp \Delta_{1}^{M} \leq 2$. Indeed, $\sharp \Delta_{1}^{M}>2$ would allow us to repeat the above construction with a new choice of $h$ so as to obtain a different Martin representation of the constant function 1 . This would contradict the uniqueness of Martin representations.

Hence, to prove that $\sharp \Delta_{1}^{M}=2$, it suffices to prove that $\sharp \Delta_{1}^{M} \geq 2$. We will show that there is a nonconstant, bounded, and positive harmonic function on $\Omega$. Then Proposition 1 yields that $\sharp \Delta_{1}^{M} \geq 2$. We are thus reduced to finding such a nonconstant function. Towards this end, recall that $F$ admits a Green's function. Hence there exists a continuous function $u$ on $\bar{D}_{1}$ such that $u$ is harmonic on $D_{1}$, $u=0$ on $\partial D_{1} \cap F$ and $u(\infty)=1$. Set

$$
v(z)= \begin{cases}u(z) & \text { for } z \in D_{1} \\ -u(\phi(z)) & \text { for } z \in D_{2}\end{cases}
$$

Then, by the reflection principle, we find that $v$ is a nonconstant bounded harmonic function on $\Omega \backslash\{-1,1\}$ with $|v|<1$ on $\Omega$. Since $\{-1,1\}$ is a polar set, the function $w=v+1$ is a nonconstant bounded, positive harmonic function on $\Omega$. The desired result follows.

Example 2. Let $F=\mathbf{R}^{3}, R=\left\{\left(x_{1}, x_{2}, 0\right): \max _{1 \leq j \leq 2}\left|x_{j}\right| \leq 1\right\}$, and define $D=F \backslash R$. Take two copies $D_{j}, j=1,2$ of $D$. Joining the upper (resp. lower) face of $R$ in $D_{1}$ to the lower (resp. upper) face of $R$ in $D_{2}$, we obtain a 2-sheeted unlimited covering space $\Omega$ of $D$. 
Let $\pi$ be the projection from $\Omega$ onto the base space $F$. Set

$$
\begin{aligned}
& E_{1}=\bigcup_{j=1}^{2}\left\{\left(x_{1}, x_{2}, 0\right):\left|x_{j}\right|=1,\left|x_{k}\right|<1 \text { for } k \neq j\right\}, \\
& E_{2}=\left\{\left(x_{1}, x_{2}, 0\right):\left|x_{1}\right|=\left|x_{2}\right|=1\right\},
\end{aligned}
$$

and $E=E_{1} \cup E_{2}$. For $z \in \Omega$ set

$$
\rho(z)= \begin{cases}\frac{1}{2} \min \{|\pi(z)-x|: x \in E\}, & \text { for } z \in \Omega \backslash \pi^{-1}(E) \\ \frac{1}{2} \min \left\{|\pi(z)-x|: x \in E_{2}\right\}, & \text { for } z \in \pi^{-1}\left(E_{1}\right) \\ 1 / 2, & \text { for } z \in \pi^{-1}\left(E_{2}\right)\end{cases}
$$

and

$$
U(z)= \begin{cases}\text { the component of } \pi^{-1}\left(B_{\rho(z)}(\pi(z))\right) \text { containing } z, & \text { for } z \in \Omega \backslash \pi^{-1}(E), \\ \pi^{-1}\left(B_{\rho(z)}(\pi(z))\right), & \text { for } z \in \pi^{-1}(E),\end{cases}
$$

where $B_{\rho(z)}(\pi(z))$ is the usual ball in $\mathbf{R}^{n}$, with center $\pi(z)$ and radius $\rho(z)$.

In Appendix, we construct charts $\varphi_{z}$ and a uniformly elliptic second order differential operator $L$ of divergence form so that $L$ is the usual Laplacian on $\varphi_{z}(U(z))$ for $z \in \Omega \backslash \pi^{-1}(E)$.

Choose $\mathcal{H}$ to be the class of solutions in open subsets of $\Omega$ for the operator $L$ on $\Omega$. We claim that $(\Omega, \mathcal{H})$ is a $\mathcal{P}$-Brelot harmonic space with a countable base, and that the minimal Martin boundary $\Delta_{1}^{M}$ of $\Omega$ consists of exactly two points.

First we check that $(\Omega, \mathcal{H})$ is a $\mathcal{P}$-Brelot harmonic space. Axiom 1 is clear. Regarding Axiom 2, recall that outside $\pi^{-1}(E), L$ is the usual Laplacian. Let $z \in \pi^{-1}(E)$. Then $L$ is a second order differential operator in divergence form and uniformly elliptic on $\varphi_{z}(U(z))$. Set $U^{\prime}(z)=\pi^{-1}\left(B_{\rho(z) / 2}(\pi(z))\right)$. By [10] (cf. [4, 6]), for any continuous function $f$ on $\partial U^{\prime}(z)$, there exists a weak solution $u \in W_{\text {loc }}^{1,2}\left(U^{\prime}(z)\right)$ to $L u=0$ on $U^{\prime}(z)$ so that $\left.u\right|_{\partial U^{\prime}(z)}=f$. Here the Sobolev space is with respect to the usual $n$-dimensional Lebesgue measure. Moreover, if $f \geq 0$ on $\partial U^{\prime}(z)$, then $u \geq 0$ on $U^{\prime}(z)$. By the Riesz representation theorem, we obtain a harmonic measure $\omega_{x}^{U^{\prime}}(z)$ $\left(x \in U^{\prime}(z)\right)$ such that, for $f \in C\left(\partial U^{\prime}(z)\right)$ the function $v(x):=\int_{\partial U^{\prime}(z)} f d \omega_{x}^{U^{\prime}(z)}$ is a weak solution to $L u=0$, continuous up to the boundary of $U^{\prime}(z)$, and satisfies $\left.v\right|_{\partial U^{\prime}(z)}=f$ on $\partial U^{\prime}(z)$. If $z \in \pi^{-1}\left(E_{2}\right)$ and $\left|z_{1}\right|=\left|z_{2}\right|=1$, we may repeat the argument above. Thus Axiom 2 follows.

By the famous Moser theorem ([15], cf. $[4,6])$, the Harnack inequality holds for non-negative functions in $\mathcal{H}$. Axiom 3 follows from this fact.

Since $\Omega$ is a covering surface on $F$ and $F$ admits Green's function $G_{z}$ with a pole $z \in F \backslash \overline{B_{2}(0)}$, we find that $G_{z} \circ \pi$ is a non-negative superharmonic function on $\Omega \backslash E$. We remark that $\pi^{-1}(E)$ is identified with $E$. Since $\varphi_{z^{\prime}}\left(E \cap U\left(z^{\prime}\right)\right)$ is a polar set in $\varphi_{z^{\prime}}\left(U\left(z^{\prime}\right)\right)$ for each $z^{\prime} \in E$ (cf. [6, Theorem 2.26]), by [1, (iv) p. 116] $G_{z} \circ \pi \circ \varphi_{z^{\prime}}^{-1}$ extends to a superharmonic function on $\varphi_{z^{\prime}}\left(U\left(z^{\prime}\right)\right)$ for each $z^{\prime} \in E$. Hence $G_{z} \circ \pi$ extends to a superharmonic function on $\Omega$. Suppose that a non-negative harmonic function $u$ satisfies $u \leq G_{z} \circ \pi$ on $\Omega$. Set $w=(u+u \circ \phi) / 2$. Clearly $w \leq G_{z} \circ \pi$ on $\Omega$. In order to prove that $u=0$ on $\Omega$, it suffices to show that $w=0$. Towards this end, notice that for each $z^{\prime} \in E, E \cap B_{\rho\left(z^{\prime}\right)}\left(z^{\prime}\right)$ is a polar set in $B_{\rho\left(z^{\prime}\right)}\left(z^{\prime}\right)$ (cf. [6, Theorem 2.26]). Since $w$ is bounded on $B_{\rho\left(z^{\prime}\right)}\left(z^{\prime}\right)$, by [1, (iv) p. 116], $w$ extends to a harmonic function on $B_{\rho\left(z^{\prime}\right)}\left(z^{\prime}\right)$ for every $z^{\prime} \in E$. Hence $w$ is a non-negative harmonic 
function on $F$ and $w \leq G_{z}$ on $F$. Since $G_{z}$ is a potential on $F$, we conclude that $w=0$. Consequently, $u=0$ on $\Omega$. Hence $G_{z} \circ \pi$ is a potential on $\Omega$.

We conclude that $(\Omega, \mathcal{H})$ is $\mathcal{P}$-Brelot harmonic space with a countable base.

It remains to be checked that $\Delta_{1}^{M}$ consists of exactly two points. Since $F$ does not admit nontrivial non-negative harmonic functions but admits a Green's function, and $E$ is a polar set, the argument at the end of Example 1 applies verbatim to verify that $\Delta_{1}^{M}$ consists of exactly two points.

Example 3. Let $F=\mathbf{R}^{2}$, and view the interval $I=[-1,1]$ as a subset of $F$. Set $D=F \backslash I$, and pick two copies $D_{j}, j=1,2$ of $D$. Joining the upper (resp. lower) edge of $I$ on $D_{1}$ to the lower (resp. upper) edge of $I$ on $D_{2}$, we obtain a 2-sheeted unlimited covering surface $\Omega$ of $D$.

Denote by $\pi$ the standard projection from $\Omega$ onto $F$. We introduce local coordinates as follows. Let $z$ be a point of $\Omega$. Set

$U(z)= \begin{cases}\text { the component of } \pi^{-1}\left(B_{\rho(z)}(\pi(z))\right) \text { containing } z, & \text { for } z \in \Omega \backslash \pi^{-1}(\{-1,1\}), \\ \pi^{-1}\left(B_{1 / 2}(\pi(z))\right), & \text { for } z \in \pi^{-1}(\{-1,1\}),\end{cases}$

where $\rho(z)=\frac{1}{2} \min \{|\pi(z)-1|,|\pi(z)+1|\}$ and $B_{\rho(z)}(\pi(z))$ is the usual disk with center $\pi(z)$ and radius $\rho(z)$ in $F$. We define the coordinate mapping $\varphi_{z}$ by setting

$$
\varphi_{z}(x)= \begin{cases}\pi(x)-\pi(z), x \in U(z), & \text { for } z \in \Omega \backslash \pi^{-1}(\{-1,1\}), \\ \sqrt{\pi(x)-\pi(z)}, x \in U(z), & \text { for } z \in \pi^{-1}(\{-1,1\}) .\end{cases}
$$

Fix $\epsilon>0$ and set $d \mu(x)=(1+|x|)^{\epsilon} d x$, for all $x \in F$. On $F$ we define

$$
L=\operatorname{div}\left(\left(1+|\cdot|{ }^{\epsilon} \nabla\right),\right.
$$

and for $z \in \Omega$, we define

$$
L_{\mu}=\operatorname{div}\left(\left(1+\left|\pi \circ \varphi_{z}^{-1}\right|\right)^{\epsilon} \nabla\right)
$$

on $\varphi_{z}(U(z))$. Let us check that $L_{\mu}$ is well defined.

Let $z \in \Omega$. Fix $\zeta \in U(z) \backslash\{-1,1\}$. Set $\varphi_{\zeta, z}=\varphi_{\zeta} \circ \varphi_{z}^{-1}$ and $U_{\zeta z}=U(z) \cap U(\zeta)$. For $\nu_{1} \in W_{\mathrm{loc}}^{1,2}\left(U_{\zeta z}\right)$ and $\nu_{2} \in C_{0}^{\infty}\left(U_{\zeta z}\right)$, we have that

$$
\begin{aligned}
& \int_{\varphi_{\zeta}\left(U_{\zeta z}\right)} L_{\mu}\left(\nu_{1} \circ \varphi_{\zeta}^{-1}\right) \nu_{2} \circ \varphi_{\zeta}^{-1} d y \\
& =-\int_{\varphi_{\zeta}\left(U_{\zeta z}\right)}\left(1+\left|\pi \circ \varphi_{\zeta}^{-1}\right|\right)^{\epsilon}\left\langle\nabla\left(\nu_{1} \circ \varphi_{\zeta}^{-1}\right), \nabla\left(\nu_{2} \circ \varphi_{\zeta}^{-1}\right)\right\rangle d y \\
& =-\int_{\varphi_{z}\left(U_{\zeta z}\right)}\left(1+\left|\pi \circ \varphi_{z}^{-1}\right|\right)^{\epsilon}\left\langle\nabla\left(\nu_{1} \circ \varphi_{\zeta}^{-1}\right) \circ \varphi_{\zeta, z}, \nabla\left(\nu_{2} \circ \varphi_{\zeta}^{-1}\right) \circ \varphi_{\zeta, z}\right\rangle J_{\varphi_{\zeta, z}} d x \\
& =-\int_{\varphi_{z}\left(U_{\zeta z}\right)}\left(1+\left|\pi \circ \varphi_{z}^{-1}\right|\right)^{\epsilon}\left\langle I_{\zeta, z}(x) \nabla\left(\nu_{1} \circ \varphi_{z}^{-1}\right)(x), \nabla\left(\nu_{2} \circ \varphi_{z}^{-1}\right)(x)\right\rangle J_{\varphi_{\zeta, z}}(x) d x \\
& =-\int_{\varphi_{z}\left(U_{\zeta z}\right)}\left(1+\left|\pi \circ \varphi_{z}^{-1}\right|\right)^{\epsilon}\left\langle\nabla\left(\nu_{1} \circ \varphi_{z}^{-1}\right)(x), \nabla\left(\nu_{2} \circ \varphi_{z}^{-1}\right)(x)\right\rangle d x \\
& =\int_{\varphi_{z}\left(U_{\zeta z}\right)} L_{\mu}\left(\nu_{1} \circ \varphi_{z}^{-1}\right) \nu_{2} \circ \varphi_{z}^{-1} d y
\end{aligned}
$$


where $I_{\zeta, z}(x)=\left(\varphi_{\zeta, z}^{-1}\right)^{\prime}\left(\varphi_{\zeta, z}(x)\right)\left(\varphi_{\zeta, z}^{-1}\right)^{\prime *}\left(\varphi_{\zeta, z}(x)\right)$ and we used the fact that $\varphi_{\zeta, z}$ is an analytic function.

Hence we find that $L_{\mu}$ is a well-defined second order elliptic differential operator of divergence form on $\varphi_{z}(U(z))$.

We again choose $\mathcal{H}$ to consist of (weak) solutions to $L_{\mu} u=0$ on open subsets of $\Omega$, and claim that $(\Omega, \mathcal{H})$ is a $\mathcal{P}$-Brelot harmonic space with a countable base, and that the minimal Martin boundary $\Delta_{\mu, 1}^{M}$ consists of exactly two points.

Let us first check that $(\Omega, \mathcal{H})$ is a $\mathcal{P}$-Brelot harmonic space. Axiom 1 is clear. Let us check Axiom 2. Suppose $z \in \Omega \backslash \pi^{-1}(\{-1,1\})$. Clearly, $L_{\mu}$ is a second order differential operator of divergence form and uniformly elliptic on $\varphi_{z}(U(z))$. Set $U^{\prime}(z)=\pi^{-1}\left(B_{\rho(z) / 2}(\pi(z))\right)$. By [10] (cf. [4, 6]), for any Lipschitz continuous function $f \in \partial U^{\prime}(z)$, there exists a weak solution $u \in W^{1,2}\left(U^{\prime}(z)\right)$ to $L_{\mu} u=0$ on $U^{\prime}(z)$, so that $\left.u\right|_{\partial U^{\prime}(z)}=f$. Moreover, if $f \geq 0$ on $\partial U^{\prime}(z)$, then $u \geq 0$ on $U^{\prime}(z)$. The discussion in $[9$, pp. 5-6], gives us the existence of a harmonic measure $\omega_{x}^{U^{\prime}(z)}\left(x \in U^{\prime}(z)\right)$ such that, for $f \in C\left(\partial U^{\prime}(z)\right), v(x):=\int_{\partial U^{\prime}(z)} f d \omega_{x}^{U^{\prime}(z)}$ is a weak solution on $U^{\prime}(z)$ to $L_{\mu} u=0$, continuous up to boundary of $U^{\prime}(z)$, and $\left.v\right|_{\partial U^{\prime}(z)}=f$ on $\partial U^{\prime}(z)$. For $z \in \pi^{-1}(\{-1,1\})$ we may repeat the argument above, and we conclude that Axiom 2 is satisfied.

By Moser's theorem ([15], cf. [4, 6]), for any relatively compact subdomain $G \subset \Omega$, the Harnack inequality holds on $G$ with respect to $L_{\mu}$. Axiom 3 follows from this fact and Axiom 1.

Next we prove that there exists a nonconstant positive $L$-potential on $F$. For this, we employ (weighted) nonlinear potential theory [6]. Set $w(x)=(1+|x|)^{\epsilon}$ and $\mu(E)=\int_{E} w(x) d x$. Fix $1<p<2$, and set

$$
f(x)= \begin{cases}x & \text { for }|x| \leq 1 \\ x|x|^{\gamma} & \text { for }|x|>1\end{cases}
$$

where $\gamma=\epsilon /(2-p)$. Then, $f: \mathbf{R}^{2} \rightarrow \mathbf{R}^{2}$ is a quasiconformal mapping and

$$
J_{f}^{1-p / 2}(x) \approx\left\{\begin{array}{ll}
1 & \text { for }|x| \leq 1 \\
|x|^{\epsilon} & \text { for }|x|>1
\end{array}=\max \left\{1,|x|^{\epsilon}\right\} .\right.
$$

By [6], $J_{f}^{1-p / 2}$ generates a doubling measure that supports a $p$-Poincaré inequality and, especially (by Hölder's inequality) a 2-Poincaré inequality. Since $w$ is comparable to $J_{f}^{1-p / 2}$, it easily follows that doubling and a 2-Poincaré inequality hold for $w$ as well. In the terminology of [6], this means that $w$ is 2-admissible and hence the full theory of [6] is available for us.

Let $B(r)=B_{r}(0)$ be the disk with center 0 and radius $r>0$. In order to prove the existence of a nonconstant positive $L$-potential, we first prove that there exists a non-negative superharmonic function $s$ on $F$ with respect to $L$ such that $s$ is $L$-harmonic on $F \backslash \overline{B(1)}, s=1$ on $B(r)$ and $\lim _{x \rightarrow \infty} s(x)=0$. To see this, by the statement and proof of [6, Theorem 9.22] (cf. [8]), it suffices to prove that $\operatorname{cap}_{2, \mu}\left(\overline{B(1)}, \mathbf{R}^{2}\right)>0$. Here, given an open set $G$ and a compact set $E \subset G$,

$$
\operatorname{cap}_{2, \mu}(E, G)=\inf _{v \in W(E, G)} \int_{G}|\nabla v|^{2} d \mu,
$$

where $W(E, G))=\left\{v \in C_{0}^{\infty}(G): u \geq 1\right.$ on $\left.E\right\}$. 
Set $A(r, R)=B(R) \backslash \overline{B(r)}$. By [6, Theorem 2.18], for $1<R$,

$$
\begin{aligned}
\operatorname{cap}_{2, \mu}(\overline{B(1)}, B(R)) & \geq(2 \pi)^{2}\left(\int_{A(1, R)}|x|^{-2}(1+|x|)^{-\epsilon} d x\right)^{-1} \\
& =2 \pi\left(\int_{1}^{R} r^{-2}(1+r)^{-\epsilon} r d r\right)^{-1} \\
& >2 \pi 2^{-\epsilon}\left(\int_{1}^{\infty} r^{-1-\epsilon} d r\right)^{-1}=2^{1-\epsilon} \pi \epsilon
\end{aligned}
$$

Since the lower bound is independent of $R$, we conclude that

$$
\operatorname{cap}_{2, \mu}\left(\overline{B(1)}, \mathbf{R}^{2}\right) \geq 2^{1-\epsilon} \pi \epsilon>0,
$$

and the existence of $s$ is shown.

Suppose that $u$ is a non-negative $L$-harmonic function on $F$ with $u \leq s$ on $F$. Since $\lim _{x \rightarrow \infty} s(x)=0$, the maximum principle yields that $u=0$. Thus $s$ is a $L$ potential on $F$. As in Example 2, one checks that $s$ generates a $L_{\mu}$-potential on $\Omega$. Consequently, $(\Omega, \mathcal{H})$ is a $\mathcal{P}$-Brelot harmonic space with a countable base.

Next we prove that $\Delta_{1, \mu}^{M}$ consists of exactly two points. First we prove that non-negative $L$-harmonic functions on $\mathbf{R}^{2}$ are constant functions. For this purpose we first prove that each non-negative $L$-harmonic function on $\mathbf{R}^{2}$ is bounded. Fix a $L$-harmonic function $u$ on $\mathbf{R}^{2}$. For $r>0$, write $C_{r}$ for the circle of radius $r$, centered at the origin 0. By the Harnack inequality we find that there exists a positive $\gamma(>1)$ independent of $u$ and $r$ so that

$$
\frac{1}{\gamma} u(x) \leq u(y) \leq \gamma u(x), \quad \text { for all } x, y \in C_{r} .
$$

Let $\omega_{0}^{B_{r}(0)}$ be the $L$-harmonic measure relative to $B_{r}(0)$ and 0 . Then

$$
u(0)=\int_{C_{r}} u d \omega_{0}^{B_{r}(0)} .
$$

Combining the above two estimates, we have, for any $x \in C_{r}$,

$$
\frac{1}{\gamma} u(x) \leq u(0)=\int_{C_{r}} u d \omega_{0}^{B_{r}(0)} \leq \gamma u(x) .
$$

Since $r$ is arbitrary, we have $\frac{u(0)}{\gamma} \leq u(x) \leq \gamma u(0)$, for any $x \in \mathbf{R}^{2}$.

Next, let $h$ be a minimal $L$-harmonic function on $\mathbf{R}^{2}$. By the above, we have $\frac{h(0)}{\gamma} \leq h(x)$, for any $x \in \mathbf{R}^{2}$. By minimality, there exists a positive $c$ with $\frac{h(0)}{\gamma}=c h$ on $\mathbf{R}^{2}$. Hence, every minimal $L$-harmonic function is a constant function on $\mathbf{R}^{2}$. Hence the minimal Martin boundary $\Delta_{1, \mu}^{M}\left(\mathbf{R}^{2}\right)$ of $\mathbf{R}^{2}$ consists of just one point. Thus let $\Delta_{1, \mu}^{M}\left(\mathbf{R}^{2}\right)=\{k\}$. By the Martin representation theorem (Proposition 1), for every non-negative $L$-harmonic function $u, u(z)=\int_{\Delta_{1, \mu}^{M}\left(\mathbf{R}^{2}\right)} u^{*}(h) d \omega_{z}^{\mu}(h)=u^{*}(k)$, for $z \in \mathbf{R}^{2}$. Hence each non-negative $L_{\mu}$-harmonic function on $\mathbf{R}^{2}$ is a constant function.

Since $F$ does not admit non-negative $L$-harmonic functions except for constant functions, we may repeat the corresponding argument from Example 1 to conclude that the minimal Martin boundary consists of at most two elements. 
Hence, to prove that $\sharp \Delta_{1, \mu}^{M}=2$, as in Example 1, it suffices to prove the existence of a nonconstant, bounded and positive $L_{\mu}$-harmonic function on $\Omega$. Let $x_{0}$ be a point of $I$. We easily check that, for all $0<t<1$,

$$
\frac{\operatorname{cap}_{2, \mu}\left(I \cap B\left(x_{0}, t\right), B\left(x_{0}, 2 t\right)\right)}{\operatorname{cap}_{2, \mu}\left(B\left(x_{0}, t\right), B\left(x_{0}, 2 t\right)\right)}>c_{1},
$$

where $c_{1}$ is independent of $x_{0}$.

By definition, ([6, p. 114]) $I$ is $(2, \mu)$-thick at every $x_{0} \in I$. Hence, by $[6$, Theorem 9.25], [6, Corollary 6.28] and [6, Theorem 9.20], we find that there exists a continuous function $u$ on $\overline{D_{1}}$ such that $u$ is harmonic on $\overline{D_{1}}$ with respect $L_{\mu}, u=0$ on $\partial D_{1} \cap F$ and $\lim _{x \rightarrow \infty} u(x)=1$. Setting

$$
v(z)= \begin{cases}u(z) & \text { for } z \in D_{1} \\ -u(\phi(z)) & \text { for } z \in D_{2}\end{cases}
$$

reflection principle shows that $v$ is a nonconstant bounded harmonic function on $\Omega \backslash \pi^{-1}(\{-1,1\})$ with respect to $L_{\mu}$ with $|v|<1$ on $\Omega$. As $k:=v+1$ is a nonconstant bounded and positive $L_{\mu}$-harmonic function on $\Omega$, the desired result follows.

Therefore we have the desired result.

\section{Appendix}

\section{Construction of charts and $\boldsymbol{L}$ for Example 2. Set}

$$
\begin{aligned}
& \varphi_{1}\left(x_{1}, x_{2}, x_{3}\right)=\left(x_{1}, r_{23} \cos \frac{1}{2} \theta_{23}, r_{23} \sin \frac{1}{2} \theta_{23}\right), \\
& \varphi_{2}\left(x_{1}, x_{2}, x_{3}\right)=\left(x_{1}, r_{23} \cos \left(\frac{1}{2} \theta_{23}+\pi\right), r_{23} \sin \left(\frac{1}{2} \theta_{23}+\pi\right)\right),
\end{aligned}
$$

where $r_{23}=\sqrt{x_{2}^{2}+x_{3}^{2}}$ and $\theta_{23}\left(0 \leq \theta_{23}<2 \pi\right)$ is the argument of the point $\left(x_{2}, x_{3}\right)$ in $\mathbf{R}^{2}$. Then the Jacobian determinant of $\varphi_{k}$ takes on the constant value $1 / 2$, the maximal value of the directional derivatives is 1 , and minimal one $1 / 2$, see [20].

Let $L[\tau](\tau \in \mathbf{R})$ be the linear mappings on $\mathbf{R}^{3}$ corresponding to the $3 \times 3$ matrices

$$
\left(\begin{array}{ccc}
\cos \tau & -\sin \tau & 0 \\
\sin \tau & \cos \tau & 0 \\
0 & 0 & 1
\end{array}\right)
$$

Notice that, for $z \in E, \pi^{-1}(z)$ is a singleton. Hence we identify $\pi^{-1}(z)$ for $z \in E$ with $z \in F$. For $z \in \pi^{-1}\left(E_{1}\right)$ with $\left|z_{i}\right|=1$ (resp. $z \in \pi^{-1}\left(E_{2}\right)$ with $\left|z_{1}\right|=\left|z_{2}\right|=1$, by choosing an appropriate $\alpha=\alpha(z)$ (resp. $\beta=\beta(z)$ ), we may assume that the argument of $\left(x_{1}, x_{2}\right)$ with $x=\left(x_{1}, x_{2}, 0\right) \in L[\alpha]\left(U(z) \cap D_{k}\right)$ (resp. of $\left(x_{1}, x_{2}\right)$ for $L[\beta]\left(U(z) \cap D_{k}\right)$ ) is between $\pi$ (resp. $\pi / 2$ ) and $2 \pi$. We assume this in what follows, unless otherwise stated.

Set

$$
\psi_{0}\left(x_{1}, x_{2}, x_{3}\right)= \begin{cases}\left(r_{12} \cos 2 \theta_{12}, r_{12} \sin 2 \theta_{12}, x_{3}\right), & 0 \leq \theta_{12}<\pi / 2, \\ \left(r_{12} \cos \frac{2}{3}\left(\theta_{12}+\pi\right), r_{12} \sin \frac{2}{3}\left(\theta_{12}+\pi\right), x_{3}\right), & \pi / 2 \leq \theta_{12}<2 \pi\end{cases}
$$

where $r_{12}=\sqrt{x_{1}^{2}+x_{2}^{2}}$ and $\theta_{12}\left(0 \leq \theta_{12}<2 \pi\right)$ is the argument of the point $\left(x_{1}, x_{2}\right)$ of $\mathbf{R}^{2}$. Then, for $0 \leq \theta_{12}<\pi / 2$ (resp. $\pi / 2 \leq \theta_{12}<2 \pi$ ) the Jacobian determinant 
of $\psi_{0}$ takes on the constant value 2 (resp. 2/3), the maximal value of the directional derivatives is 2 (resp. 1), and minimal one 1 (resp. 2/3), see [20].

For $z \notin E_{3}$, we define the coordinate mapping $\varphi_{z}$ by

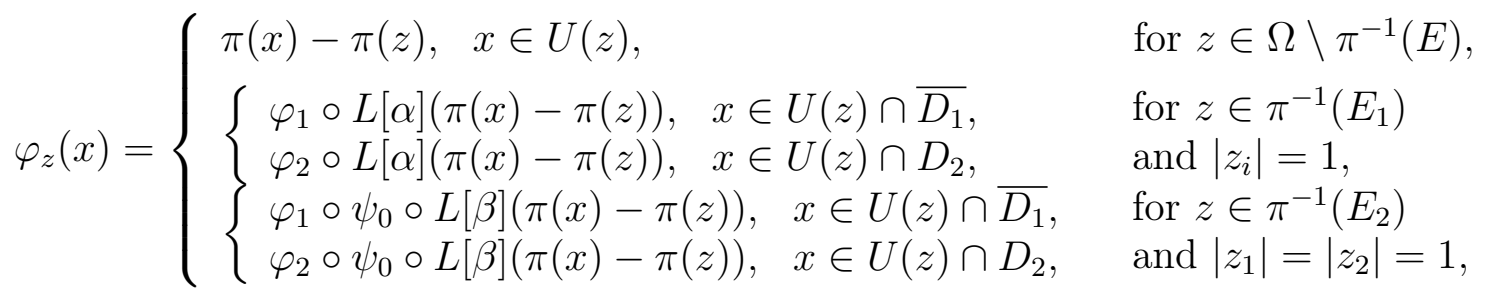

where $\overline{D_{1}}$ is the closure of $D_{1}$ in the sense of $\Omega$.

We introduce a second order differential operator $L=L_{z}$ of elliptic type on $\Omega$ by setting

$$
L=\operatorname{div}\left\{\phi_{z} \phi_{z}^{*} \nabla\right\} \quad \text { on } \varphi_{z}(U(z)), \quad \text { for } z \in \Omega,
$$

where

$$
\phi_{z}= \begin{cases}I_{3}, & \text { for } z \in \Omega \backslash \pi^{-1}(E), \\
& \begin{array}{ll}
\sqrt{2} \varphi_{1}^{\prime}(L[\alpha](\pi(x)-\pi(z))), & x \in U(z) \cap \overline{D_{1}}, \\
\sqrt{2} \varphi_{2}^{\prime}(L[\alpha](\pi(x)-\pi(z))), & x \in U(z) \cap D_{2}, \\
\text { for } z \in \pi^{-1}\left(E_{1}\right) \text { and }\left|z_{i}\right|=1, & \\
\sqrt{3} \varphi_{1}^{\prime}\left(\psi_{0} \circ L[\beta](\pi(x)-\pi(z))\right) \psi_{0}^{\prime}(L[\beta](\pi(x)-\pi(z))), & x \in U(z) \cap \overline{D_{1}}, \\
\sqrt{3} \varphi_{2}^{\prime}\left(\psi_{0} \circ L[\beta](\pi(x)-\pi(z))\right) \psi_{0}^{\prime}(L[\beta](\pi(x)-\pi(z))), & x \in U(z) \cap D_{2}, \\
\text { for } z \in \pi^{-1}\left(E_{2}\right) \text { and }\left|z_{1}\right|=\left|z_{2}\right|=1 . &
\end{array}\end{cases}
$$

Above, we used the notation $\psi^{\prime}$ for the differential matrix of a mapping $\psi$.

We claim that $L$ is a well-defined second order differential operator of divergence form and uniformly elliptic on $\varphi_{z}(U(z))$ for $z \in \Omega$.

Indeed, for $z \in \Omega \backslash \pi^{-1}(E), L$ is the usual Laplacian on $\varphi_{z}(U(z))$. Let $z \in \pi^{-1}(E)$. First we consider $z \in \pi^{-1}\left(E_{1}\right)$. Suppose that $\left|z_{i}\right|=1$. Set $\varphi_{\zeta z}=\varphi_{\zeta} \circ\left(\varphi_{z}\right)^{-1}$ $(\zeta \in U(z))$ and set $U_{\zeta z}=U(z) \cap U(\zeta)$. Then

$$
\varphi_{\zeta z}(y)= \begin{cases}\left(\varphi_{1} \circ L[\alpha]\right)^{-1}(y)+\pi(z)-\pi(\zeta), & y \in \varphi_{z}\left(U_{\zeta z} \cap \overline{D_{1}}\right), \\ \left(\varphi_{2} \circ L[\alpha]\right)^{-1}(y)+\pi(z)-\pi(\zeta), & y \in \varphi_{z}\left(U_{\zeta z} \cap D_{2}\right) .\end{cases}
$$

Since the eigenvalues of $\left(\varphi_{k}^{-1}\right)^{\prime}(k=1,2)$ consist of 1 and 2 , the Jacobian determinants $J_{\left(\varphi_{k}\right)^{-1}}$ of $\left(\varphi_{k}\right)^{-1}$ both take on the constant value 2 on $\varphi_{z}\left(U(z) \cap \overline{D_{k}}\right)(k=1,2)$, and $L[\alpha]$ is an orthonormal matrix, we find that $1 \leq\left|\left(\varphi_{\zeta z}\right)^{\prime}(v)\right| \leq 2$ when $|v|=1$. For $\nu_{1} \in C^{\infty}\left(\varphi_{\zeta}\left(U_{\zeta z}\right)\right)$ and $\nu_{2} \in C_{0}^{\infty}\left(\varphi_{\zeta}\left(U_{\zeta z}\right)\right)$, we have that

$$
\begin{aligned}
\int_{\varphi_{\zeta}\left(U_{\zeta z}\right)} \nu_{2} \Delta \nu_{1} d y & =-\int_{\varphi_{\zeta}\left(U_{\zeta z}\right)}\left\langle\nabla \nu_{1}, \nabla \nu_{2}\right\rangle d y \\
& =-\int_{\varphi_{z}\left(U_{\zeta z}\right)}\left\langle\left(\nabla \nu_{1}\right) \circ \varphi_{\zeta z},\left(\nabla \nu_{2}\right) \circ \varphi_{\zeta z}\right\rangle J_{\varphi_{\zeta z}} d x \\
& =-2 \int_{\varphi_{z}\left(U_{\zeta z}\right)}\left\langle\left(\varphi_{\zeta z}^{-1}\right)^{\prime} \circ \varphi_{\zeta z}\left(\varphi_{\zeta z}^{-1}\right)^{\prime *} \circ \varphi_{\zeta z} \nabla\left(\nu_{1} \circ \varphi_{\zeta z}\right), \nabla\left(\nu_{2} \circ \varphi_{\zeta z}\right)\right\rangle d x \\
& =2 \int_{\varphi_{z}\left(U_{\zeta z}\right)} \operatorname{div}\left\{\left(\varphi_{\zeta z}^{-1}\right)^{\prime} \circ \varphi_{\zeta z}\left(\varphi_{\zeta z}^{-1}\right)^{\prime *} \circ \varphi_{\zeta z} \nabla\left(\nu_{1} \circ \varphi_{\zeta z}\right)\right\} \nu_{2} \circ \varphi_{\zeta z} d x
\end{aligned}
$$


A simple calculation shows that $2\left(\varphi_{\zeta z}^{-1}\right)^{\prime} \circ \varphi_{\zeta z}\left(\varphi_{\zeta z}^{-1}\right)^{\prime *} \circ \varphi_{\zeta z}=\phi_{z} \phi_{z}^{*}$. Hence $L$ is well defined. Since $1 / 2 \leq\left|\left(\varphi_{\zeta z}^{-1}\right)^{\prime} v\right| \leq 1$ when $|v|=1$, we find that $L_{z}$ is a second order differential operator of divergence form and uniformly elliptic on $\varphi_{z}(U(z))$. For $z \in \pi^{-1}\left(E_{2}\right)$, by the similar argument to that for $z \in \pi^{-1}\left(E_{1}\right)$ we see that $L$ is well defined and $L$ is a second order differential operator of divergence form and uniformly elliptic on $\varphi_{z}(U(z))$.

Proof of Reflection Principle. Let $x_{0}$ be a point of the open interval $(-1,1)$. Take a positive number $\rho$ with $x_{0}-\rho, x_{0}+\rho \in[-1,1]$. Set $C=\partial B\left(x_{0}, \rho\right), C_{+}=$ $C \cap\left\{\left(x_{1}, x_{2}\right): x_{2} \geq 0\right\}$ and $C_{-}=C \cap\left\{\left(x_{1}, x_{2}\right): x_{2} \leq 0\right\}$. Set $\gamma_{+}=C_{+} \cup\left[x_{0}-\rho, x_{0}+\rho\right]$ and $\gamma_{-}=C_{-} \cup\left[x_{0}-\rho, x_{0}+\rho\right]$.

Set

$$
v b_{+}(z)= \begin{cases}u(z), & \text { for } z \in C_{+}, \\ 0, & \text { for } z \in C_{-},\end{cases}
$$

and

$$
v b_{-}(z)= \begin{cases}0, & \text { for } z \in C_{+}, \\ -u(z), & \text { for } z \in C_{-} .\end{cases}
$$

Let $v_{+}$(resp. $v_{-}$) be the generalized Dirichlet solution of $v b_{+}$(resp. $\left.v b_{-}\right)$. Then $v_{+}+v_{-}=0$ on $\left[x_{0}-\rho, x_{0}+\rho\right]$. Denote by $F_{+}$(resp. $\left.F_{-}\right)$the bounded domain surrounded by $\gamma_{+}$(resp. $\left.\gamma_{-}\right)$. By this fact and the minimum principle $u=v_{+}+v_{-}$ on $F_{+}$and $-u=v_{+}+v_{-}$on $F_{-}$. Hence $v=v_{+}+v_{-}$.

Therefore we have the desired result.

\section{References}

[1] Brelot, M.: Lectures on potential theory. - Lectures on Mathematics 19, Tata Institute of Fundamental Research, Bombay, 1960.

[2] Constantinescu, C., and A. Cornea: Ideale Ränder Riemannscher Flächen. - Ergeb. Math. Grenzgeb. (2) 32, Springer-Verlag, Berlin-Göttingen-Heidelberg, 1963.

[3] Constantinescu, C., and A. Cornea: Potential theory on harmonic spaces. - Grundlehren Math. Wiss. 158, Springer-Verlag, New York-Heidelberg, 1972.

[4] Gilbarg, D., and N. S. Trudinger: Elliptic partial differential equations of second order. Classics Math., Springer, 1998.

[5] Gowrisankaran, K.: Fatou-Naïm-Doob limit theorems in the axiomatic system of Brelot. - Ann. Inst. Fourier (Grenoble) 16, 1966, 455-467.

[6] Heinonen, J., T. Kilpeläinen, and O. Martio: Nonlinear potential theory of degenerate elliptic equations. - Dover, 2006.

[7] Hervé, R. M.: Recherches axiomatiques sur la theorie des fonctions surharmoniques et du potentiel. - Ann. Inst. Fourier (Grenoble) 12, 1962, 415-571.

[8] Holopainen, I., and P. Koskela: Volume growth and parabolicity. - Proc. Amer. Math. Soc. 129, 2001, 3425-3435.

[9] Kenig, C. E.: Harmonic analytic techniques for second order elliptic boundary value problems. - CBMS Reg. Conf. Ser. Math. 83, Amer. Math. Soc., Providence, RI, 1994.

[10] Littman, W., G. Stampaccia, and H. F. Weinberger: Regular points for elliptic equations with discontinuous coefficients. - Ann. Sc. Norm. Super. Pisa Cl. Sci. (3) 17, 1963, 43-77.

[11] Masaoka, H.: When do the harmonic Hardy spaces with distinct indices coincide on a hyperbolic Riemann surface? - Acta Human. Sci. Univ. Sangio Kyotiensys 37, 2008, 1-9. 
[12] Masaoka, H., and S. Segawa: Hyperbolic Riemann surfaces without unbounded positive harmonic functions. - Adv. Stud. Pure Math. 44, 2006, 227-232.

[13] Masaoka, H., and S. Segawa: On several classes of harmonic functions on a hyperbolic Riemann surface. - Complex analysis and its applications, OCAMI Stud. 2, Osaka Munic. Univ. Press, Osaka, 2007, 289-294.

[14] McKean, H. P., and D. Sullivan: Brownian motion and harmonic functions on the class surface of the thrice punctured sphere. - Adv. in Math. 51, 1984, 203-211.

[15] Moser, J.: On Harnack's theorem for elliptic differential equations. - Comm. Pure Appl. Math. 14, 1961, 577-591.

[16] NAÏM, L.: $\mathcal{H}^{p}$-spaces of harmonic functions. - Ann. Inst. Fourier (Grenoble) 17, 1967, 425-469.

[17] SARIo, L., and M. NAKAI: Classification theory of Riemann surfaces. - Grundlehren Math. Wiss. 164, Springer-Verlag, New York-Berlin, 1970.

[18] Schiff, J. L.: $H^{p}$-Spaces of harmonic functions and the Wiener compactification. - Math. Z. 132, 1973, 135-140.

[19] TôKI, Y.: On the examples in the classification of open Riemann surfaces. I. - Osaka Math. J. 5, 1953, 267-280.

[20] VÄISÄLÄ, J.: Lectures on $n$-Dimensional quasiconformal mappings. - Lecture Notes in Math. 229, Springer-Verlag, 1971.

Received 23 April 2012 • Accepted 7 September 2012 